\title{
Predicting acute liver failure in dengue infection
}

\author{
R S Kumarasena ${ }^{1}$, M A Niriella ${ }^{2}$, C K Ranawaka ${ }^{1}$, J K N D Miththinda ${ }^{1}$, A P De Silva ${ }^{2}$, A S Dassanayaka ${ }^{2}$, \\ H J de Silva ${ }^{2}$
}

(Index words: dengue infection, hepatic dysfunction, acute liver failure, prediction)

\begin{abstract}
In a retrospective study involving 240 patients with dengue infection, we attempted to identify early predictors of acute liver failure (ALF). Sixteen out of 41 patients with serum AST more than $1000 \mathrm{IU} / \mathrm{ml}$ developed ALF compared to none with serum AST less than $1000 \mathrm{IU} / \mathrm{ml}$. Among patients with serum AST more than $1000 \mathrm{IU} / \mathrm{ml}$, presence of two of the three following phenomena, within the first 5 days of illness: elevated serum bilirubin, elevated alkaline phosphatise or persistent nausea and vomiting, predicted development of ALF (93.8\% sensitivity, 98.7\% specificity, $83.3 \%$ positive predictive and $99 \%$ negative predictive value). The presence of elevated serum bilirubin, alkaline phosphatase and persistent nausea and vomiting in patients with very high serum AST during the early phase of dengue infection should alert the physician of impending ALF.
\end{abstract}

Ceylon Medical Journal 2016; 61: 35-36

DOI: http://doi.org/10.4038/cmj.v61i1.8260

\section{Introduction}

Dengue virus infection (DI) is endemic in Sri Lanka. Organ specific complications such as myocarditis and acute liver failure (ALF) occur more frequently now. Hepatic dysfunction in DI is attributed either to a direct viral effect on hepatocytes or a dysregulated host immune response to the virus. Infection with dengue serotypes DENV 2, 1 and 3, respectively, correlate with decreasing severity of liver damage as evidenced by elevation of transaminases [1]. Asymptomatic elevation of aspartate transaminase (AST) and alanine transaminase (ALT) is common in DI. However, DI may also cause hepatic injury of severity comparable with other types of viral hepatitis. Although the true incidence is not known, dengue associated ALF is not uncommon and has a poor prognosis
[2, 3]. Early identification of impending ALF in DI will help clinicians to identify patients who will benefit from interventions such as intravenous $\mathrm{N}$-acetyl cysteine (NAC) [4]. The objective of this study was to identify early clinical and biochemical predictors of ALF in DI.

\section{Methods}

In a retrospective cohort study we attempted to identify early predictors of ALF in patients admitted to hospital with DI confirmed by dengue IgM antibodies detected by ELISA technique on day six or later. As ours is a tertiary referral centre for liver disease, DI patients included both direct admissions as well as referrals with severely deranged liver functions. All patients admitted between 1 January 2009 and 31 March 2010 with confirmed DI, and had complete data were included in the analysis. Demographic details, clinical course and serial blood results were extracted from patient records. For the purpose of the study clinical features and biochemistry within the first five days of illness were considered as potential early predicators of ALF. The 2009 World Health Organisation dengue classification was used and ALF was defined using American Association for Study of Liver Diseases criteria: presence of coagulation abnormality (INR $\geq 1.5$ ) and any degree of mental alteration (encephalopathy) in a patient without pre-existing liver disease and an illness of less than 26 weeks duration [5].

Given the possibility of only a small percentage of patients with DI developing ALF, a general linear model was used to identify the potential early risk factors. Approval for the study was obtained from the Ethics Review Committee of the Faculty of Medicine, University of Kelaniya.

\section{Results}

Data of 240 patients with DI [57.7\% male; mean age 35.6 years (SD 15.4)] were available for analysis. All patients

${ }^{1}$ University Medical Unit, Colombo North Teaching Hospital, Ragama and ${ }^{2}$ Faculty of Medicine, University of Kelaniya, Ragama, Sri Lanka.

Correspondence: MAN, e-mail: <maduniln@yahoo.co.uk>. Received 8 October and revised version accepted 12 December 2015. 
had been admitted within the first five days of illness. Severe DI was seen in 49/240 (20.4\%). Fifteen of them (15/ 49) had hypotensive shock. ALF developed in 16 (32.6\%) patients with severe DI. Only 4/16 patients with ALF had hypotensive shock. Abdominal pain, persistent nausea and vomiting (PNV), bleeding, hepatomegaly and ascites were present in 125 (52.1\%), 92 (38.3\%), 39 (16.2\%), 129 (53.8\%) and 28 (11.7\%) patients respectively. Elevated AST, serum bilirubin (SB), alkaline phosphatase (AP) and gamma glutamyl transpeptide (GGT) were observed in 208 (86.7\%), 20 (8.3\%), 18 (7.5\%) and 60 (25\%) patients respectively.

Of the 240 patients, 41 (17.1\%) had serum AST more than $1000 \mathrm{IU} / \mathrm{ml}$, and $16 / 41$ patients with serum AST more than $1000 \mathrm{IU} / \mathrm{ml}$ developed ALF (6.7\%) compared to none with serum AST less than $1000 \mathrm{IU} / \mathrm{ml}$. A general linear model showed that PNV $(p<0.001)$, elevated SB $(p<0.001)$ and elevated AP ( $p=0.004)$ within the first five days of the illness predicted ALF in patients with serum AST more than $1000 \mathrm{IU} / \mathrm{ml}$. Presence of two of these three factors could predict ALF with a 93.8\% sensitivity (CI 69.7-99.0\%), 98.7\% specificity (CI 96.1-99.7), 83.3\% positive predictive value (CI 58.6-96.2\%) and 99\% negative predictive value (CI 97.5-99.9\%).

\section{Discussion}

The case fatality rate in DI with associated ALF has been reported to be as high as $50 \%$ [3]. The mortality in ALF does not depend on the severity of dengue infection as fatality rates were similar in those with and without shock [3]. In the present study only $25 \%$ of patients who developed ALF had hypotensive shock. A previous study has shown that transaminases and alkaline phosphatase are significantly higher in patients with severe dengue, haemorrhage, sequential infection and non-survivors, and that high SB, transaminases and AP are markers of poor prognosis [6]. Our results seem to confirm these findings. We evaluated clinical and biochemical parameters within the first five days of the illness in order to investigate factors that may predict ALF early in the course of the illness.

Liver transplantation, which is the definitive treatment for ALF, is not available in most countries where dengue is prevalent. Even if liver transplantation is possible, there are many technical difficulties in dengue patients due to bleeding, hypotension and multi-organ failure. Intravenous NAC improves transplant-free survival in early stage non-acetaminophen acute liver failure. Several case reports and case series suggest early use of NAC many improve survival in dengue associated ALF [4]. Early prediction and recognition of ALF in DI may, therefore, be helpful to identify patients who may benefit from treatment with intravenous NAC.

The retrospective nature of this study is a major limitation. Findings of our study should be considered preliminary and needs confirmation by larger prospective studies. Furthermore, given the small number of patients who developed ALF, it was not possible to use logistical regression model and instead a general linear model was used.

In conclusion, results of our study suggest that the presence of elevated SB, elevated AP and PNV in patients with very high serum AST levels during the early phase of DI should alert physicians to the possibility of impending ALF.

\section{Conflicts of interests}

There are no conflicts of interest.

\section{References}

1. Seneviratne SL, Malavige GN, de Silva HJ. Pathogenesis of liver involvement during dengue viral infections. Trans $R$ Soc Trop Med Hyg 2006; 100: 608-14.

2. Chung-Huang Kuo CH, Dar-in Tai, Chi-Sin Chang-Chien, et al. Liver biochemical tests and dengue fever. Am J Trop Med Hyg 1992; 47: 265-70.

3. Chongsrisawat V, Hutagalung $Y$ and Poovorawan Y. Liver function test results and outcomes in children with acute liver failure due to dengue infection. Southeast Asian J Trop Med Public Health 2009; 40: 47.

4. Kumarasena RS, Senanayake M, Sivaraman K, et al. Intravenous N-Acetylcysteine in dengue-associated acute liver failure. Hepatol Int 2010; 4: 533-4.

5. Dengue: Guidelines for Diagnosis, Treatment, Prevention and Control TDR/WHO, Geneva, Switzerland, 2009.

6. Chhina RS, Goyal O, Chhina D K, et al. Liver function tests in patients with dengue viral infection. Dengue Bulletin 2008; 32: 110-7. 